Rapid Reviews COVID-19•

\title{
Review 2: "Modelling the effectiveness and social costs of daily lateral flow antigen tests versus quarantine in preventing onward transmission of COVID-19 from traced contacts"
}

\section{Timothy Russell ${ }^{1}$}

1"London School of Hygiene \& Tropical Medicine Infectious Disease Epidemiology UNITED KINGDOM"

Published on: Nov 10, 2021

License: Creative Commons Attribution 4.0 International License(CC-BY 4.0). 


\section{$\underline{\text { RR:C19 Evidence Scale rating by reviewer: }}$}

- Strong. The main study claims are very well-justified by the data and analytic methods used. There is little room for doubt that the study produced has very similar results and conclusions as compared with the hypothetical ideal study. The study's main claims should be considered conclusive and actionable without reservation.

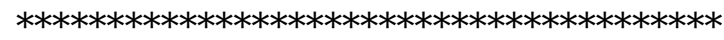

\section{Review:}

\section{Summary}

The study developed a detailed composite model of COVID-19 transmission dynamics by combining three submodels: one of the individual-level viral load trajectories; one describing the timing of the onset of symptoms for individuals; and lastly, a model of the onwards infectiousness of each individual. The paper extends the models in Larremore et al. (2020) and Kissler et al. (2021) by including each step of the infection process at the individual level. The three subcomponents of the model are relatively simple and digestible when considered on their own. The overall model, with its many parameters, assumptions and priors, starts to become unwieldy-as is the case with many detailed infectious disease models!

The work attempts to answer questions that remain very important and underaddressed. Specifically, whether the complex picture of daily testing routines outperforms that of blanket quarantine requirements (which are still in place in many countries around the world). Clearly, quarantine has knock-on negative effects and it would be in many countries' interests to reduce such effects. However, robust results around the issue are very hard to come by; given that modelling, even the infectious disease consequences of such strategies (let alone the socioeconomic effects) are very challenging. I believe the paper does a fantastic job of attempting to answer such questions and the conclusions are in line with similar results. Overall, the work is of very high quality, the modelling seems well thought through with sensible parametric choices for each subcomponent (exponential growth/decline for viral load dynamics and Hill functions for onward infectiousness, both of which are relatively standard choices), and with reasonable fixed values or priors were appropriate and fit in a Bayesian hierarchical framework. Lastly, the text is clear and well-written. I especially liked the second last paragraph in the introduction, which very nicely outlined the 
challenges with this sort of work-specifically discussing why existing work often underestimates the effectiveness of repeated testing with lateral flow tests. I also think the result of the cumulative sensitivity of repeated LFAs is comparable to a PCR test. I think this is one of the key messages of the paper. However, I do have some major comments that I believe need to be addressed before the work is of publishable standard.

\section{Major comments}

- When I saw the difference between the relative infectiousness curves inferred using the Lee et al. (2021) data and the paired contact tracing data, I was expecting large differences between the inferred results that rely on those curves. However, the incubation period, the generation time, and the time until the onset of symptoms distributions are very similar for both of the relative infectiousness curves. The text mentions that the model was calibrated so these distributions reflect the realistic (empirical) estimates for the same distributions. I believe the authors could elaborate on this calibration and exactly why the results are so robust to the markedly different relative infectiousness curves, as it seems important that the procedure undertaken includes realistic parameters.

- Many of the results presented do not have explicit uncertainty. All of the figures in the main text do not contain uncertainty. The supplementary information goes through in detail the hierarchical Bayesian inference model used (implemented in stan and rstan). As such, it is not particularly difficult to include uncertainty (in the form of a highest density interval or even credible interval). Would it be possible to include the uncertainty at least for Figures 1 and 2? Figures 3 and 4 would become very messy and adherence is varied across a wide range, making uncertainty a bit less informative. I believe it is important to show the uncertainty on the estimates in some form, even if it is just with supplementary figures and keeping the main figures without uncertainty.

- The codebase for the work does not seem to be available anywhere. This is somewhat subjective, but I believe at least a minimum working example of the codebase should be shared (possibly with simulated data if the data is not publicly available) for detailed modelling studies such as this. It is important for transparency, but also for reusability for others. It is difficult to give a detailed review of a modelling paper without being able to see any of the underlying codebase. 
- I believe the supplementary material would greatly benefit from a summary table for all the model parameters. Tables are included in the different model subsections for estimated parameters, but the tables just summarise the inferred posteriors. A table with all of the model parameters, which of the submodels they are used in, a description of the parameter and whether they are fixed or estimated (if estimated, then state the chosen prior/priors) would help the reader visualise the model as a whole.

\section{Minor comments}

- The text labels in Figure 3 (coming off of the individual lines) overlap a bit-making them difficult to distinguish in places. Perhaps the authors could use a legend instead or resize the scale in the vaccinated panel to separate them somewhat.

- Lastly, this is a very minor point, but would it be possible to provide examples of countries that have successfully implemented some of the suggested strategies discussed in the final paragraph of the discussion (to strengthen the point)? 